\title{
ESTUDO DA PERDA DE CARGA NO ESCOAMENTO DE FLUIDO NÃO NEWTONIANO EM COILED TUBING
}

\author{
B. C. LEAL $^{1}$, B. R. OLIVEIRA ${ }^{1}$, L. P. FILHO ${ }^{1}$, L. A. CALÇADA ${ }^{1}$, C. M. SCHEID ${ }^{1}$. \\ ${ }^{1}$ Universidade Federal Rural do Rio de Janeiro, Departamento de Engenharia Química \\ Contato: barbaraleal.eq@outlook.com
}

\begin{abstract}
RESUMO - O coiled tubing, também chamado de flexitubo, é composto por um tubo de metal flexível e contínuo que é enrolado em um carretel com várias camadas. No processo de fechamento de poços de petróleo, o tubo é desenrolado em direção ao poço por onde diferentes tipos de fluido são bombeados, como a pasta de cimento que é um fluido não newtoniano. É de vital importância prever as condições de processo para se obter a perda de carga e a pressão de bombeio adequada para escoamento do fluido. O objetivo deste trabalho é avaliar experimentalmente a perda de carga no escoamento em coiled tubing para diferentes vazões e, então, propor um modelo que ajuste os dados experimentais. Desta maneira, experimentos foram conduzidos em uma unidade piloto bombeando água e solução de goma xantana em diferentes vazões e razões de curvatura, que é razão entre os raios de bobina do carretel e da camada. Uma metodologia para o cálculo da perda de carga em coiled tubing foi proposta utilizando-se correlações de fator de atrito presentes na literatura. Estas correlações levam em conta fatores como a razão de curvatura da bobina, diâmetro e comprimento do tubo enrolado e a velocidade do fluido em cada camada, fatores estes que podem variar em um caso real. Dessa forma, será possível prever a pressão de bombeio necessária para garantir o escoamento do fluido. Foi possível propor um modelo baseado em correlações da literatura, com erros menores que $5 \%$.
\end{abstract}

\section{INTRODUÇÃO}

O coiled tubing é a expressão em inglês para tubos enrolados, ou flexitubos, que designa um tubo de metal flexível e contínuo que é enrolado em um carretel com várias camadas (Figura 1). Os tubos enrolados podem ser fabricados em diversos materiais e tamanhos, resultando nas mais variadas aplicações como microreatores, trocadores de calor, turbinas, itens de plataforma, e no caso do presente estudo, a cimentação do poço após seu tempo de utilização. Por ser uma tubulação contínua, não necessita de conexões como os drill pipes que precisam ser rosqueados para alcançar o poço. Outra facilidade frente aos drill pipes é que o carretel torna a unidade muito mais compacta e fácil de transportar. Essas características mostram o quanto o uso deste sistema é vantajoso, pois reduzindo maquinário, tempo e espaço, consequentemente reduz-se o custo operacional de um processo. A Figura 2 mostra o coiled tubing da unidade experimental usada neste estudo. 
Figura 1 - Coiled tubing e injetor.

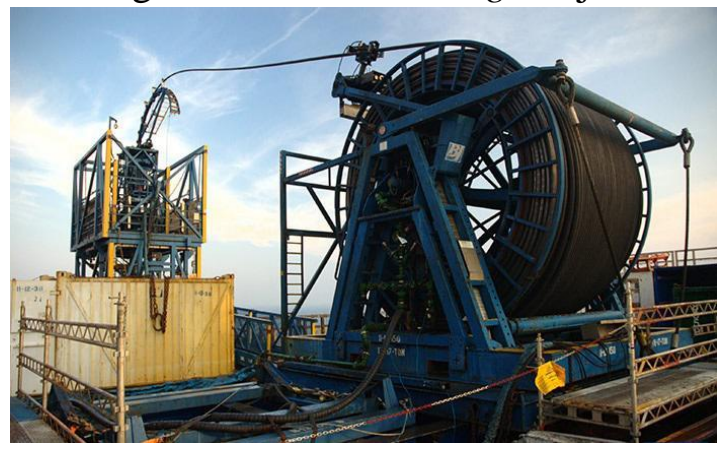

Figura 2 - Unidade experimental.

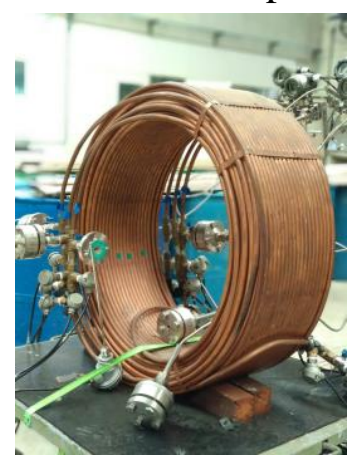

Segundo Fox e McDonald (1994), os fluidos não newtonianos são caracterizados por não apresentarem uma relação linear da tensão de cisalhamento com a taxa de deformação, ou seja, não seguem a Lei de Newton da viscosidade. A pasta de cimento possui comportamento de fluido pseudoplástico, cuja viscosidade aparente decresce com o aumento da taxa de deformação. O modelo Power-Law (Equação 1) descreve o comportamento reológico da pasta de cimento.

$\tau=k \gamma^{n}$

Onde: $\tau$ é a tensão de cisalhamento $\left(\mathrm{g} / \mathrm{cm} . \mathrm{s}^{2}\right)$, $\mathrm{k}$ é o índice de consistência do fluido $\left(\mathrm{Pas}^{\mathrm{n}}\right)$, $\gamma$ é a taxa de cisalhamento $\left(\mathrm{s}^{-1}\right)$ e n é o índice de comportamento.

A perda de carga pode ser definida como a perda de energia dinâmica do fluido ao escoar. A intensidade dessa perda é influenciada tanto pela tubulação em que o escoamento ocorre, quanto pelas características do fluido e das condições operacionais. Correlações na literatura descrevem o fator de atrito para escoamento de fluidos não newtonianos em tubos curvados, como Mashelkar e Devarajan (1977) apud Zhou e Shah (2004b), Mishra e Gupta (1979) e McCann et al. (1996). O objetivo deste estudo é validar a correlação que, associada ao modelo proposto, descreva o fenômeno. A correlação de Mishra e Gupta (1979) é apresentada na Equação 2 com suas respectivas condições nas equações 3 a 6 .

$f_{c}=f_{r}\left[1+0,033(\log D e)^{4}\right]$

$f_{r}=\frac{16}{R e}$

$\mu_{a p}=K\left(\frac{8\langle v\rangle}{D}\right)^{n-1}$

$R e=\frac{D<v>\rho}{\mu_{a p}}$

$D e=\operatorname{Re}\left(\frac{r}{R}\right)^{0,5}$

Na Equação 7 tem-se a correlação de McCann et al. (1996), com suas respectivas condições nas equações 8 e 9 . 
$f_{c}=\frac{1,06 a}{R e^{0,8 b}}\left(\frac{r}{R}\right)^{0,1}$

$a=\frac{\log (n)+3,93}{50}$

$b=\frac{1,75-\log (n)}{7}$

Onde: $\mathrm{f}_{\mathrm{c}}$ é o fator de atrito para tubo curvado (adimensional), $\mathrm{f}_{\mathrm{r}}$ é o fator de atrito para tubo reto (adimensional), De é o número de Dean (adimensional), Re é o número de Reynolds (adimensional), D é o diâmetro do tubo $(\mathrm{m}),\langle v\rangle$ é a velocidade média $(\mathrm{m} / \mathrm{s}), \rho$ é a massa específica $\left(\mathrm{kg} / \mathrm{m}^{3}\right), \mu$ é a viscosidade aparente $(\mathrm{kg} / \mathrm{ms}), \mathrm{r} / \mathrm{R}$ é a razão de curvatura (razão entre raios da bobina e da camada), a e b são parâmetros do modelo de McCann (1996).

\section{MATERIAIS E MÉTODOS}

A unidade experimental utilizada é composta por uma bobina com 8 camadas de tubos de cobre com 1/2 in de diâmetro, totalizando $375,8 \mathrm{~m}$ de extensão (Figura 2). Foram instalados termopares e transdutores de pressão a cada camada a fim de se avaliar a perda de carga. Um sistema de bombeio foi acoplado a unidade experimental. $\mathrm{O}$ fluido é preparado em um tanque de 200 L que possui um sistema de agitação e uma camisa para o controle de temperatura. $\mathrm{O}$ controle de temperatura é feito por resistências e um chiller com precisão de 0,2 a $0,3{ }^{\circ} \mathrm{C}$. $\mathrm{O}$ fluido é bombeado para a linha com uma bomba de deslocamento positivo de $25 \mathrm{HP}$, onde a vazão de escoamento é controlada por um inversor de frequência e medida por um medidor de vazão. Ao sair do tanque, o fluido é direcionado ao coiled tubing e retorna ao tanque escoando em um circuito fechado. Os resultados são aquisitados através de um Controlador Lógico Programável (PLC), acoplado a um software projetado para esta unidade, que registra todos os parâmetros avaliados nos intervalos de tempo desejados ao experimento. Foram também realizados experimentos a fim de comparar o comportamento reológico da goma frente a água no sistema coiled tubing. Estes experimentos adotaram a mesma metodologia citada anteriormente, observando como a perda de carga varia com a vazão. O esquema da unidade é apresentado na Figura 3.

Figura 3 - Esquema da unidade experimental.

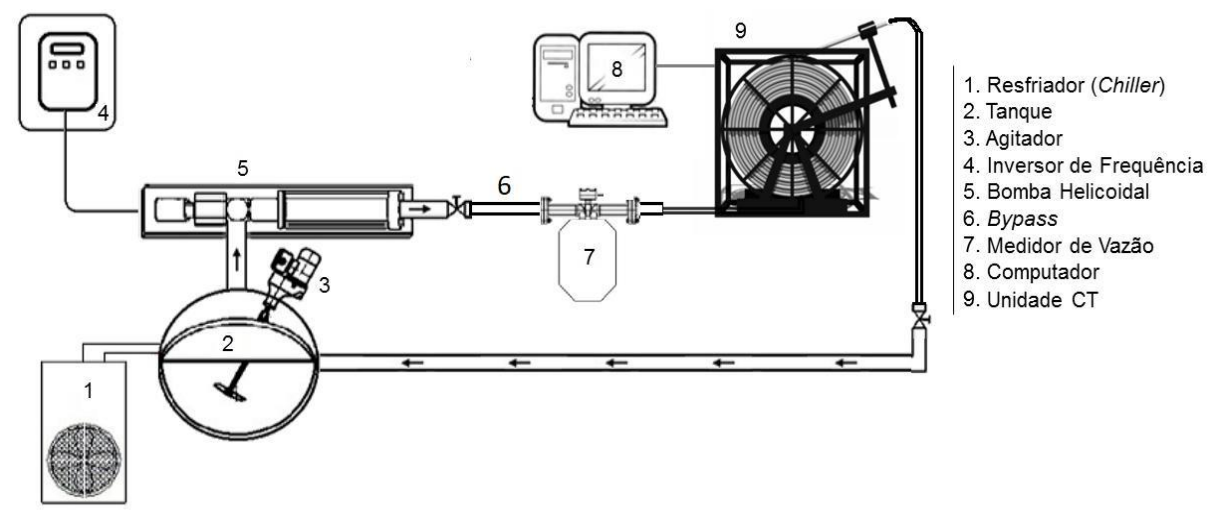

A fim de se avaliar a perda de carga no escoamento de um fluido não-newtoniano em tubo curvado, uma metodologia experimental foi adotada para posterior validação de correlações de fator de atrito. Os experimentos foram realizados utilizando uma solução de goma xantana 
com concentração de $2 \mathrm{lb} / \mathrm{bbl}$ visando um comportamento semelhante ao da pasta de cimento. Esta concentração foi escolhida após um estudo reológico utilizando-se diferentes concentrações visando um comportamento semelhante ao da pasta de cimento. Com o inversor de frequência ligado à bomba, variou-se a vazão volumétrica de 0,50 a $2,0 \mathrm{~m} / \mathrm{h}$ e medidas de pressão foram obtidas a cada camada, em triplicata, com a temperatura constante. Para o cálculo do fator de atrito experimental, a equação de Bernoulli (Equação 10) para fluidos incompressíveis e reais escoando em regime permanente foi aplicada, onde considerou-se o sistema sem fornecimento ou retirada de energia na forma de trabalho. Além disso, as alturas dos pontos de entrada e saída foram consideradas iguais.

$\frac{P_{1}}{\rho g}+\frac{\left\langle v_{1}\right\rangle^{2}}{2 g}+z_{1}-h_{t}=\frac{P_{2}}{\rho g}+\frac{\left\langle v_{2}\right\rangle^{2}}{2 g}+z_{2}$

$h_{t}=h_{s}+h_{d}$

Simplificando 10 e 11, obtém-se 12.

$h_{d}=4 f \frac{L}{D} \frac{\langle v\rangle^{2}}{2 g}$

A perda de carga distribuída pode ser escrita segundo Fanning (Fox e McDonald, 1994) pela Equação 13.

$h_{d}=\frac{\Delta P}{\rho g}$

Reunindo as Equações 12 e 13 obtém-se a Equação para fator de atrito experimental, dada por 14.

$f=\frac{\Delta P D}{2 \rho L\langle v\rangle^{2}}$

Onde: $\mathrm{P}_{\mathrm{i}}$ são as pressões no ponto $\mathrm{i}(\mathrm{Pa}), \mathrm{g}$ é a gravidade $\left(\mathrm{m} / \mathrm{s}^{-1}\right), \mathrm{z}_{\mathrm{i}}$ é a altura no ponto $\mathrm{i}$ $(\mathrm{m}),\langle v\rangle$ é a velocidade média no ponto $\mathrm{i}\left(\mathrm{m} / \mathrm{s}^{-1}\right), \mathrm{h}_{\mathrm{t}}$ é a perda de carga total $(\mathrm{m}), \mathrm{h}_{\mathrm{d}}$ é a perda de carga distribuída $(\mathrm{m}), \mathrm{h}_{\mathrm{s}}$ é a perda de carga localizada $(\mathrm{m}), \Delta \mathrm{P}$ é a diferença entre pressões $\left(\mathrm{N} / \mathrm{m}^{2}\right)$, L é o comprimento do tubo (m), f é o fator de atrito (adimensional).

A partir do cálculo de fator de atrito experimental foi possível validar a correlação de fator de atrito dada pelas equações 2 e 7 . A avaliação foi feita por meio da comparação entre os valores de perda de carga experimental e os calculados, para diferentes vazões e razões de curvatura. Como cada camada tem uma razão de curvatura e consequentemente um fator de atrito diferente, optou-se por calcular a perda de carga total como uma soma das perdas de carga em cada camada (Equação 15). A modelagem foi conduzida em linguagem FORTRAN.

$$
\left(\frac{\Delta P}{\rho g}\right)=\left(2 f_{1} \frac{L_{1}}{D} \frac{v^{2}}{g}\right)_{1^{\mathbf{a}} \mathrm{cam} .}+\left(2 f_{2} \frac{L_{2}}{D} \frac{v^{2}}{g}\right)_{2^{\mathrm{a}} \mathrm{cam} .}+\cdots+\left(2 f_{n} \frac{L_{n}}{D} \frac{v^{2}}{g}\right)_{n \text {-ésima cam. }}
$$




\section{RESULTADOS}

A fim de se comparar a perda de carga de fluidos newtonianos e não-newtonianos em coiled tubing, a Figura 4 apresenta o gráfico de perda de carga em toda a extensão do tubo em função da vazão de água e goma xantana, com as barras de erro dos pontos experimentais.

Figura 4 - Perda de carga total em função da vazão de água e goma xantana.

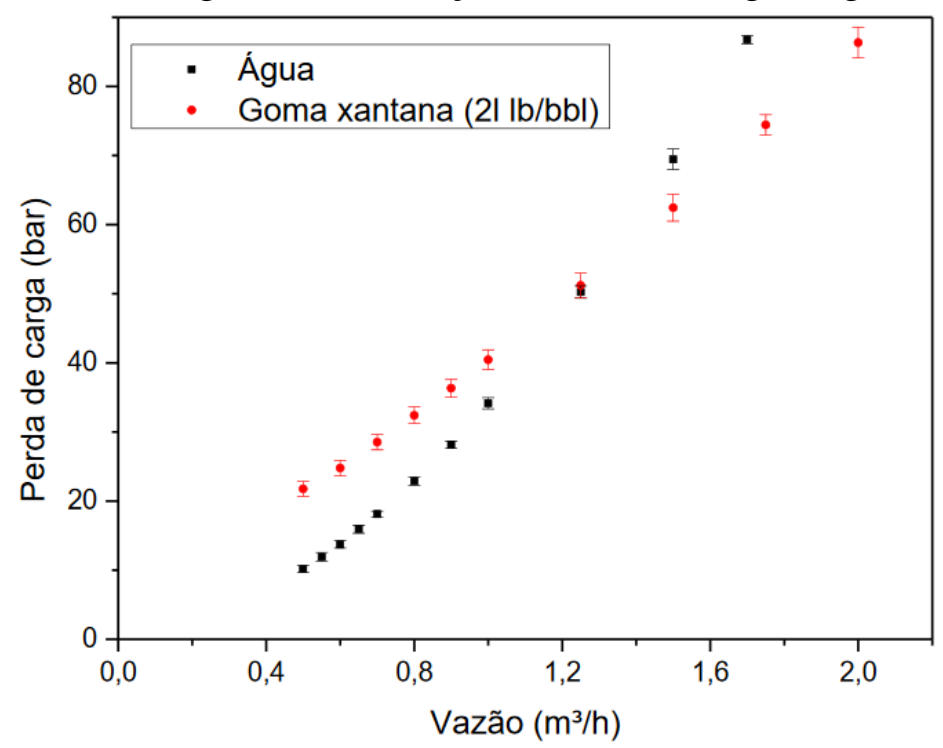

Observa-se que em vazões mais baixas, o escoamento com água apresenta menores valores de perda de carga em relação a solução de goma xantana. Entretanto, a partir de aproximadamente $1,25 \mathrm{~m}^{3} / \mathrm{h}$, esta relação se inverte. Ou seja, a solução de goma passa a ter menores perdas de carga. Este resultado pode ser explicado pelo comportamento reológico da goma xantana. Com o aumento da vazão e consequentemente da taxa de cisalhamento, a viscosidade aparente da solução diminui, reduzindo a resistência ao fluxo e a perda de carga (Fox e McDonald, 1996).

A Figura 5 apresenta os resultados de perda de carga como uma função da vazão previstos com o auxílio das correlações de Mirsha e Gupta (1979) e McCann (1996). Pode-se observar que os resultados não aderem aos dados experimentais. Com base neste fato, uma nova correlação de fator de atrito foi proposta baseada na correlação de Mirsha e Gupta (1979), adicionando um fator de ajuste. O modelo é apresentado na Equação 16, mantendo as condições das equações 4 a 6 .

$$
f_{c}=f_{r}\left[1+0,033\left(\log D e_{2}\right)^{4}\right] 0,67
$$

Pode-se observar, pela Figura 5, o melhor ajuste dos resultados gerados pela nova correlação com os dados experimentais, visto que a mesma se encontra dentro das barras de erro dos pontos experimentais. 
Figura 5 - Perda de carga de goma xantana em função da vazão utilizando correlações da literatura e o modelo de ajuste proposto.

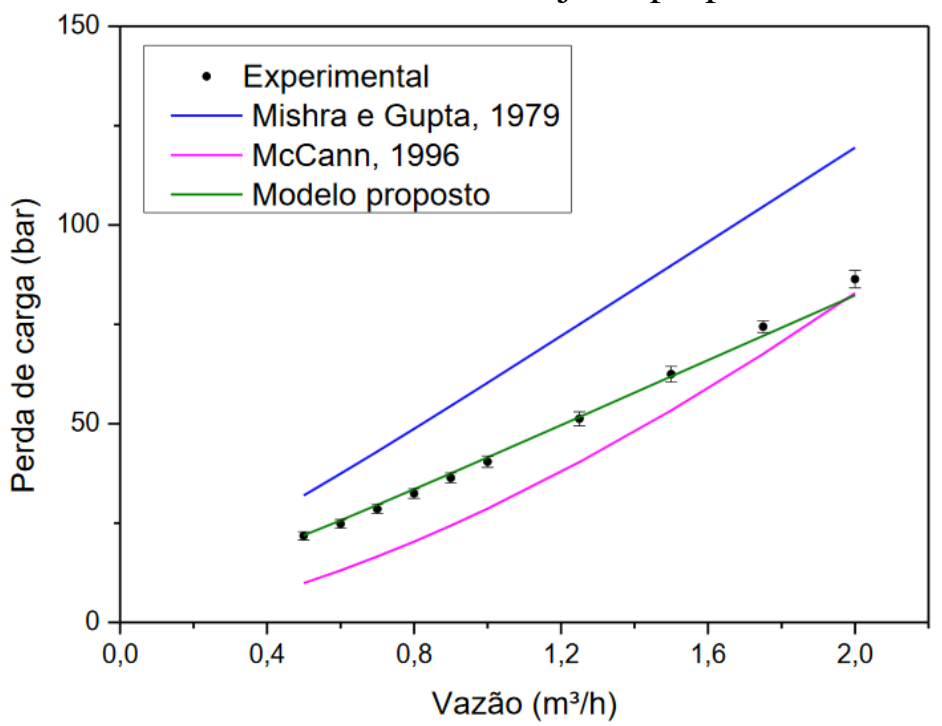

Na tabela 1 são apresentados os valores de perda de carga total obtidos para a correlação proposta e seus respectivos erros relativos para cada vazão. Pode-se observar que foi possível prever a perda de carga em função da vazão e da razão de curvatura com erros relativos abaixo de $5 \%$.

Tabela 1 - Resultados de perda de carga para cada vazão no modelo e seus erros relativos

\begin{tabular}{|c|c|c|c|c|c|c|c|c|c|c|}
\hline $\mathrm{Q}^{\left(\mathrm{m}^{3} / \mathrm{h}\right)}$ & 0,50 & 0,60 & 0,70 & 0,80 & 0,90 & 1,0 & 1,25 & 1,50 & 1,75 & 2,00 \\
\hline$\Delta \mathrm{P}_{\exp }($ bar $)$ & 21,75 & 24,78 & 28,53 & 32,43 & 36,34 & 40,45 & 51,21 & 62,46 & 74,42 & 86,36 \\
\hline$\Delta \mathrm{P}_{\text {calc }}($ bar $)$ & 21,90 & 25,69 & 29,57 & 33,52 & 37,50 & 41,53 & 51,68 & 61,88 & 72,12 & 82,31 \\
\hline Erro $(\%)$ & $-0,70$ & $-3,68$ & $-3,65$ & $-3,37$ & $-3,22$ & $-2,67$ & $-0,92$ & 0,90 & 3,10 & 4,68 \\
\hline
\end{tabular}

\section{CONCLUSÃO}

A partir deste estudo foi possível prever a perda de carga para fluidos não newtonianos em coiled tubing através da proposta de um modelo baseado em correlações da literatura. A validação dessa correlação de fator de atrito foi feita a partir de experimentos conduzidos em uma unidade piloto. O erro percentual foi inferior a cinco, o que corrobora o uso desta correlação. Logo, pode ser utilizada para predizer a perda de carga no escoamento de pasta de cimento, visto que a reologia deste fluido também pode ser descrita pelo modelo reológico utilizado para solução de goma xantana.

\section{REFERÊNCIAS BIBLIOGRÁFICAS}

FOX, Robert W.; MCDONALD, Alan T. Introduction to Fluid Mechanics, John Wiley \& Sons. Inc., New York, 1994.

MCCANN, R. C. et al. Frictional pressure loss during turbulent flow in coiled tubing. In: SPE Gulf Coast Section/ICoTA North American Coiled Tubing Roundtable. Society of Petroleum Engineers, 1996.

MISHRA, P.; GUPTA, S. N. Momentum transfer in curved pipes.2. Non-Newtonian fluids. Industrial \& Engineering Chemistry Process Design and Development, v.18, n.1, p.137-142,1979.

ZHOU, Y. et al. Fluid flow in coiled tubing: a literature review and experimental investigation. Journal of Canadian Petroleum Technology, v. 43, n. 06, 2004. 